\title{
Erratum to: The IEA-EEF European Congress of Epidemiology 2012: Epidemiology for a Fair and Healthy Society
}

\author{
Portuguese Association of Epidemiology
}

Published online: 2 November 2012

(C) Springer Science+Business Media Dordrecht 2012

\section{Erratum to: Eur J Epidemiol (2012) 27:S1-S197 DOI 10.1007/s10654-012-9722-6}

Due to a technical error, this accepted abstract was not included in the supplement "The IEA-EEF European Congress of Epidemiology 2012 Epidemiology for a Fair and Healthy Society". The conference organizers apologize for the inconvenience.

\section{Conceptual-theoretical Model for the Determinants of the Excess of Caesarean Sections in the Private Healthcare Sector in Brazil}

\section{Jacqueline Torres, Maria do Carmo Leal, Rosa Domingues}

Escola Nacional de Saúde Pública Sérgio Arouca, ENSP/ FIOCRUZ, Agência Nacional de Saúde Suplementar

Background In Brazil, approximately 46 million people use the private healthcare sector. The $\mathrm{C}$-section prevalence in this sector is around $90 \%$. It is a public health problem, since scientific evidence shows that the isolated effect of birth via $\mathrm{C}$-section increases the risk of maternal and neonatal morbidity and mortality. There are no reports of other countries with such a high prevalence of $\mathrm{C}$-section and thus suggesting the existence of local contextual determinants in the causal chain of this problem. Studying causal links of the excess of

The online version of the original article can be found under doi:10.1007/s10654-012-9722-6.

Portuguese Association of Epidemiology ( $\square)$

Portugal

e-mail: ape@ape.org.pt
C-sections in the private healthcare sector in Brazil, demands a priori, the elaboration of a conceptual-theoretical model that enables the proper connection between theoretical and empirical levels in epidemiological research in this area, thereby contributing to a better selection of variables, consequently adding value to the validity of studies.

Objectives To indicate causal constructs and to present a conceptual-theoretical model for the excess in C-sections performed in the private healthcare sector in Brazil, from the policy-makers' point of view.

Methods An exploratory study, which used qualitative approach through face-to-face interviews with 9 policymakers, who are responsible for the development of strategies in order to reduce $\mathrm{C}$-sections in Brazil. Excerpts from the interviews which mentioned the determinants for the excess of C-sections were selected, grouped and summarized in dimensions, with subsequent identification of causal constructs for this problem. The counting of the frequency of citation from such causal constructs has allowed its relevant classification. For the validation of the findings, each policy-maker received his/her interview transcript and causal dimensions that were extracted from the interview, and they were questioned whether it reflected the view expressed by the interviewee. The theoreticalconceptual model was developed, using identified causal constructs, by a panel of experts.

Outcomes Nine causal constructs were identified- "Medical Convenience"; "Caesarean Section: the Best Choice"; "Autonomy in Obstetrics"; "Maternity-hotel"; "Tangential Regulation"; "Inadequate Training"; "Childbirth Care as a Physician Action"; "Delegated Responsibility" and "Changes in Obstetric Risk Profile". The first 4 were identified as the most relevant in determining the problem. The theoretical-conceptual model highlights the urgency of factors related to the management of childbirth care, to the 
detriment of clinical factors, in the causal chain of excess $\mathrm{C}$-sections in the private healthcare sector in Brazil.

Conclusions Epidemiological studies which aim to identify determinants for the excess of $\mathrm{C}$-sections in the private healthcare sector in Brazil should include variables related to the care pathway. 